$5^{\text {th }}$ International Conference on Biotechnology Applications in Agriculture (ICBAA), Benha University, 8 April 2021, Egypt (Conference Online)

\title{
Soil Quality as Indicated by Physical and Chemical Properties in Some Tanta Areas, Nile Delta, Egypt Using Remote Sensing and GIS Techniques.
}

\author{
Maha A. M. Abdel-razek, Heba. S. Rashed, Mohamed. A. Abdel-salam and Mohamed H.H. Abbas \\ Department of Soil and Water, Faculty of Agricultural, Moshtohor, Benha Universty, Egypt.
}

Corresponding Author: maha.abdelrazek@fagr.bu.edu.eg

\begin{abstract}
The present study aimed at assessing the physical and chemical quality of the soils in Tanta area of El-Gharbia Governorate, Egypt. Its area is 33760 ha (located between longitudes $30^{\circ} 45^{\prime}$ and $31^{\circ} 20^{\prime} \mathrm{E}$ and latitudes $30^{\circ} 35^{\prime}$ and $\left.31^{\circ} 15^{\prime} \mathrm{N}\right)$. The mapping units in the study area: overflow basin (OB), high river terrace (RT1), moderate river terrace (RT2), low river terrace (RT3). Nine soil profiles were selected to represent Tanta area soils in addition to 18 soil samples. Twelve physio-chemical parameters which used to assess soil quality were chosen to evaluate soil quality including; texture (T), drainage (D), effective depth $(\mathrm{P})$, parent materials $(\mathrm{M})$, rock fragment $(\mathrm{R})$, slope gradient $(\mathrm{S})$, hydraulic conductivity $(\mathrm{H})$, water holding capacity $(\mathrm{W})$, electrical conductivity $(\mathrm{C})$, soil reaction $(\mathrm{O})$, exchangeable sodium percentage $(\mathrm{G})$ and calcium carbonate $(\mathrm{N})$. Results showed that Tanta district could be classified into one class according to the physical quality measures (moderate), while results of chemical quality index revealed two classes (high and moderate). Over $44 \%$ of the soil of Tanta area is of moderate soil quality index while $55.51 \%$ is of low soil quality index according to both physical and chemical parameters. The low soil quality dominates the areas characterized by shallow depth, poor drainage and hydraulic conductivity. The results demonstrated that management of soil practices should be carefully associated with soil characteristics.
\end{abstract}

Keywords: Soil quality, Tanta district, Nile Delta, Remote sensing and GIS.

\section{Introduction}

Egypt is one of the most old countries worldwide. It is located geographically in the northeastern corner of Africa (between latitudes of $22^{\circ}$ and $32^{\circ} \mathrm{N}$ and longitudes $25^{\circ}$ and $37^{\circ} \mathrm{E}$ ) (Zahran and Willis, 2009; Negm et al., 2017 and Embabi, 2018). The Nile Delta in Egypt, with its fringes, covers an area of 22,000 $\mathrm{km}^{2}$. The Nile Delta was formed during flood seasons by Nile sediments during the Late Miocene as an apron in the North Delta embayment. Its sediments are coarse, derived from the elevated Tertiary rocks of the Eastern Desert. Sediments of the ancient Niles, called the Paleonile, Prenile, and Neonile, cover wide tracts along the eastern and western margins of the delta (Said 1981). The oldest sedimentary rocks penetrated in the Nile Delta are the shallow marine Late Jurassic carbonates, which are overlain un conformably by the Early Cretaceous sediments that interbedded carbonate-clastic sequence un conformably underlies the earliest Tertiary sediments, which is un conformably overlain by the Late Eocene-Early Oligocene shale section (Younes, 2015). Central part of Nile Delta is classified by sedimentary nonconsolidated deposits belonging to the quaternary area that is differentiated into four different deposits: young deltaic, Fluvio-marine, young Eolian, and old Eolian (Abu-hashim, 2015). El-Asmar and Hereher (2011) and Embabi (2018) recorded that Nile Delta with its triangular shape is a nearly flat plain. Its surface slopes gently northwards, where the difference in elevation between its apex in the south and the Mediterranean coast is $+18 \mathrm{~m}$ (Sestini, 1992). According to Moustaf (2000) Geology of the Nile Delta areas is largely divided into two geologic units; Nile River alluvium and undifferentiated basement rocks. The soils are sandy texture outside the cultivated areas in the Delta, whereas very high clay content exists in this Delta producing some infertile black-alkali soils as well as saline soils (Negm, 2017). The soils of alluvial and alluvio-marine deposits contain loam and clay to clay-loam. According to Omran (2017) most of the Nile Delta soils are recent alluvial soils and most soils are originated from the ancient Nile sediments, which are mostly derived from igneous and metamorphic rocks of the Abyssinian Plateau. The major landscape in ElGharbia is the flood plain and these soils are originated from Nile sediments before High Dam construction. They are developed from sediments of Ethiopian plateau transported by Nile River and subsequently deposited in both the valley and Delta (El-Baroudy, 2015).

Land evaluation used to describe and quantify the sustainable productive capacity of land (Mackay et al., 2018), it is an integral part of land use planning, has been established as one of the preferred methods to support sustainable land use management. In essence, land evaluation aims to compare and match each potential land use with the properties of individual parcels of land, also called land units (De la Rose (2005); Palm et al. (2007); Niekerk, (2010); Liniger et al. (2011) and Govers et al. (2013). Based on Daneshvar (2017) Land suitability evaluation mainly focuses on environmental attributes that refers to the spatial, ecological and social configurations of land use development in urban planning. Hence, a multi-criteria evaluation method is used in order to find out the sustainable balance to assess the suitability index of land units for sustainable urban development (Joerin et al., 2001 and Hossain and Das 2010). 
According to Wander et al. (2002); Blum (2003); Schjonning et al. (2004) and Novak et al. (2010) Soil quality is a measure of the ability of soil to carry out particular ecological and plant productive, has interconnections with management practices, productivity and other aspects as well as human health (Doran, 2002 and Zornoza et al., 2015). The concept of soil quality integrates physical, chemical and biological properties of soil for a specific land use and an account of the soil's ability to provide ecosystem and social services through its capacities to perform its functions under changing conditions (Karlen et al., 1997; Seybold et al., 1997; Wang and Gong, 1998; Southorn and Cattle, 2004; Wienhold et al., 2004 and Shukla et al., 2006). Soil quality has gained impetus, many methods such as land use capability classification (Klingebiel and Montgomery, 1961), soil quality cards and test kits (Craig and Arlene, 2002), soil quality index method (Doran and Parkin, 1994 and Doran and Jones, 1996), dynamic variable soil quality method (Larson and Pierce, 1994), Soil Management Assessment Framework (SMAF) (Andrews et al., 2004), and Cornell Soil Health Assessment (CSHA) have been developed to determine quality scores. These two methods are used by many researchers, and their effectiveness in sustainable use of soil is evident (Karlen et al., 2008; Rashidi et al., 2010; Adeyolanu et al., 2013 and Karlen et al., 2014). Land uses and management practices have significant influences on soil quality. It is reported that differences in fertilization, cropping system and farming practices were the main factors influencing soil quality at field scale (Liu et al., 2010). Usually, factors such as excessive tillage, planting system, excessive fertilizer use, changes in land use, organic fertilizer use, and applied planting rotation directly affect soil quality (Cambardella et al., 2004; Liebig et al., 2004; Nael et al., 2004; Ozgoz et al., 2013; Yao et al., 2013; Nakajima et al., 2015 and Sacco et al., 2015). Some studies also showed tillage management and manure application are among the important factors affecting soil quality (Shirani et al., 2002 and Yang et al., 2004). Soil quality( SQ ) indicators are a composite set of measurable physical, chemical, and biological attributes which relate to functional soil processes and can be used to evaluate SQ status, as affected by management (Karlen et al., 1997;Arshad and Martin, 2002 and Allen et al., 2011). Direct measurement of the soil quality indicators is time-consuming and expensive. Soil quality assessment is essential to monitor the agricultural systems in order to maintain its sustainability (AbdelRahman and Tahoun, 2019). The agricultural soils in El-Gharbia governorate are characterized by high soil productivity depending on its chemical and physical properties (Mohamed et al., 2015). Tanta soils are within the area that was classified as Vertisols (Afify et al. 2011). Afify et al. (2008) showed that these Vertisols in Nile Delta are highly suitable for the common cultivated crops that were highly adapted with this alluvium.

The present study aimed at (i) identifying and evaluating soil quality of Tanta district in El-Gharbia Governorate depending on soil physical and chemical characteristics. (ii) Producing thematic maps of soil quality index in Tanta area for proper future planning.

\section{Materials and Methods}

\section{Location of Study Area}

Tanta district of the El-Gharbiya Governorate is located in the heart of delta midway between Damietta and Rashid between longitudes $30^{\circ} 45^{\prime}$ and $31^{\circ} 20^{\prime} \mathrm{E}$ and latitudes $30^{\circ} 35^{\prime}$ and $31^{\circ} 15^{\prime} \mathrm{N}$ (Fig. 1). It covers an area about $337.6 \mathrm{~km}^{2}$ (33760 ha) out of $1942 \mathrm{~km}^{2}$ (194200 ha) of the El-Gharbiya governorate (Belal and Moghanm, 2011; Mohamed et al., 2015; Masoud, 2016; Masoud et al., 2016 and Shokr et al., 2016). 


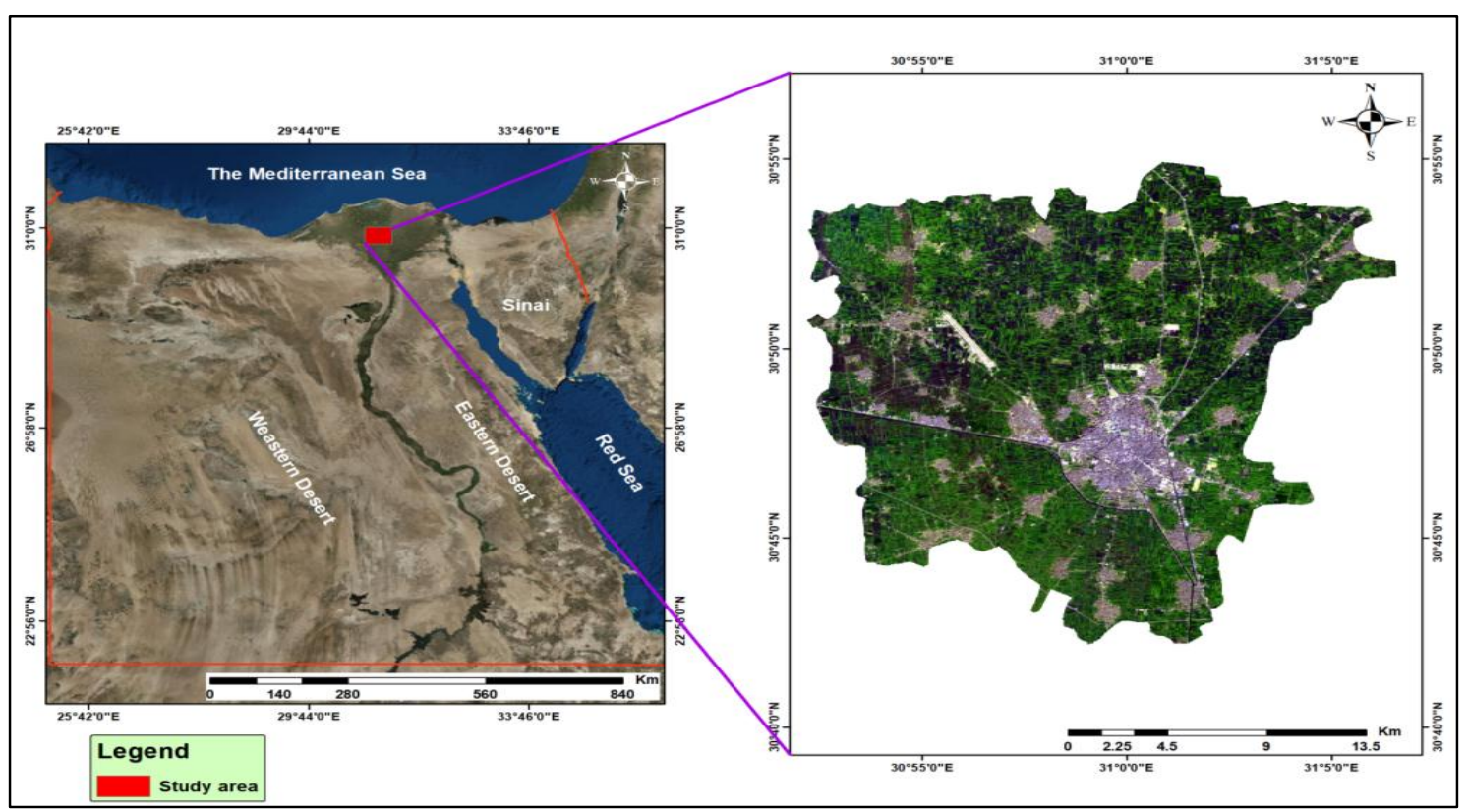

Fig. 1: Location map of the study area.

\section{Climate of study area}

The climate in the Nile Delta is generally Mediterranean with hot summers and mild winters (Zahran and Willis, 2009; Ismael, 2015 and Masoud et al., 2016). Average temperatures are $18^{0}$
$\mathrm{C}$ in winter and $31^{\circ} \mathrm{C}$ in summer. Precipitation ranges from 22 to $200 \mathrm{~mm} /$ year. Annual rainfall is $50 \mathrm{~mm}$ mostly in winter. Figure 2 shows the climatology diagram of El-Gharbia (2010-2018).

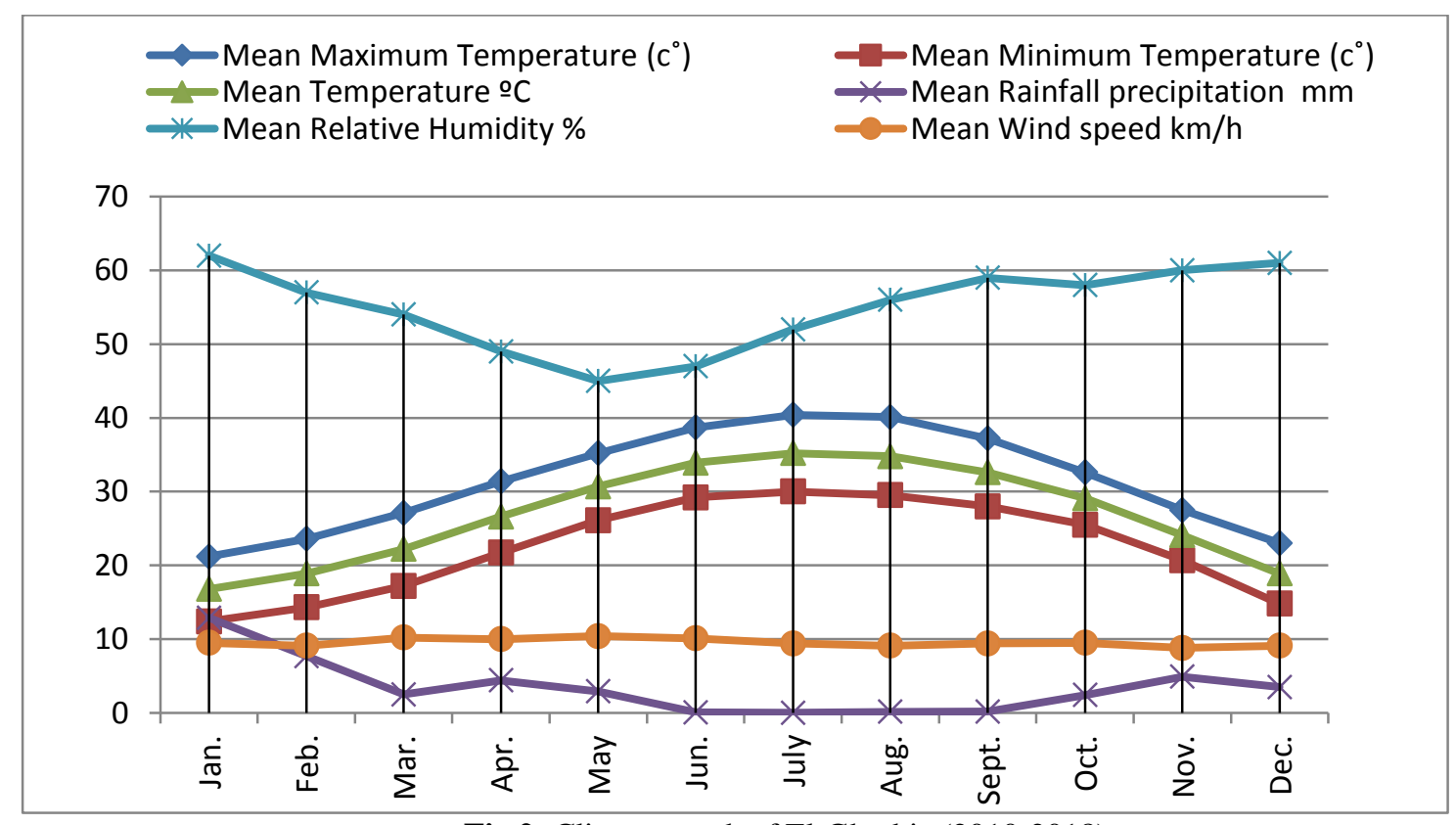

Fig.2: Climate graph of El-Gharbia (2010-2018).

\section{Geology and geomorphology of study area}

Tanta and its suburban are built on the Holocene soils made up of flat-lying alluvial plain averaging 8.5 $\mathrm{m}$ above mean sea level ranging between $11 \mathrm{~m}$ at the south and $6 \mathrm{~m}$ at its northern part. Soils are represented in the Nile delta by the Holocene Bilqas formation. This forms the top layer of the flood plain of the modern Nile made up of silty clay, brown at the top and gray in the lower part, constituting the agricultural soil of the delta (Masoud, 2016). The study area lies in Tanta and lies in a semi-arid climate zone.

Field Work and Laboratory Analysis

Based on the pre-field interpretation and information gained during the reconnaissance, eighteen samples were collected from nine profiles. Morphological Description of soil profiles, which represent the different geomorphic units were carried out according to the guidelines for soil description 
(FAO, 2006). The laboratory analyses of soil samples that collected and analyzed using the Methods of Soil, Plant and Water Analysis (Estefan et al., 2013).The analyses include, soil Samples preparation, particle size distribution, Soil color (Anon, 1975), soil pH, organic matter concentration, free $\mathrm{CaCO} 3$ content, Hydraulic conductivity, electric conductivity (dS/m), bulk Density, particle density, soil porosity, soil moisture content, gypsum content, cation exchange capacity (cmol/kg soil),exchangeable sodium percentage, available $\mathrm{N}$ and available $\mathrm{P}, \mathrm{K}, \mathrm{Fe}$, $\mathrm{Mn}, \mathrm{Zn}$, and $\mathrm{Cu}$. Using the field work and laboratory analyses data, the soils classify with the World Reference Base on USDA Soil Taxonomy(USDA, 2014).

Soil Quality Index (SQI).

The physical and chemical soil quality are determined from the indices recommended by ElNagaar et al. (2013) and calculated using the following equations:

\section{Equation of physical soil quality Index (PQI)}

The physical quality index (PQI) was estimated for the different mapping units of the study area as the following equation:

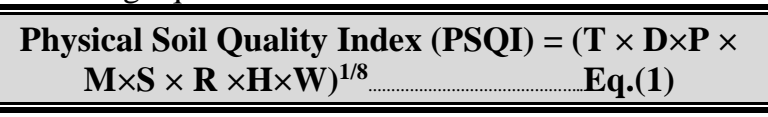

Where PSQI is the Physical Soil Quality Index, T is the texture, D is the drainage, $\mathrm{P}$ is effective depth, $\mathrm{M}$ is parent material, $\mathrm{S}$ is the slope gradient, $\mathrm{R}$ is the rock fragments, $\mathrm{H}$ is the hydraulic conductivity, and $\mathrm{W}$ is the water holding capacity. Each factor is rated on a scale from 1 to 2 , the actual percentages being multiplied by each other. The resultant is the index of quality (between 1.13 and 1.45).

\section{Equation of chemical soil quality Index (CQI)}

The chemical quality Index (CQI) was estimated for the different mapping units of the study area as the following equation:

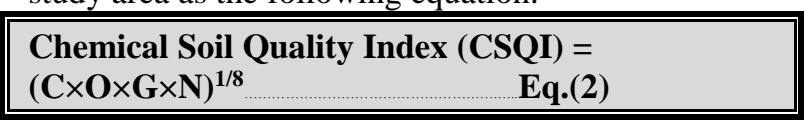

Where CSQI is the Chemical Soil Quality Index, $\mathrm{C}=$ electric conductivity $(\mathrm{EC}), \mathrm{O}=$ soil reaction $(\mathrm{pH})$, $\mathrm{G}=$ exchangeable sodium percentage (ESP), and $\mathrm{N}=$ calcium carbonate $\left(\mathrm{CaCO}_{3}\right)$. Each factor is rated on a scale from 1 to 2 , the actual percentages being multiplied by each other. The diagnostic factors of each thematic layer were assigned values of factor rating identified in Tables 1, 2,3,4,5 and 6.The rating of soil quality of the soils was done according to the grading system in Table 8 .

Table 1. Definition of texture and slope gradient

\begin{tabular}{|c|c|c|c|c|c|c|c|}
\hline \multicolumn{4}{|c|}{ Texture (T) } & \multicolumn{4}{|c|}{ Slope gradient $(\mathrm{S})$} \\
\hline Class & Texture & Description & Index & Class & $\begin{array}{c}\text { Slope } \\
\text { gradient }(\%)\end{array}$ & Description & Index \\
\hline T1 & $\begin{array}{l}\text { L, SCL, SL, } \\
\text { LS, CL }\end{array}$ & Good & 1.00 & S1 & $<6$ & Very gentle & 1.00 \\
\hline $\mathbf{T 2}$ & $\begin{array}{l}\text { SC, SiL, + } \\
\text { SiCL, }\end{array}$ & Moderate & 1.33 & $\mathbf{S 2}$ & $6-18$ & Moderately & 1.33 \\
\hline T3 & $\mathrm{Si}, \mathrm{C}, \mathrm{SiC}$ & Poor & 1.66 & S3 & $18-35$ & Steep & 1.66 \\
\hline $\mathbf{T 4}$ & $S$ & Very poor & 2.00 & S4 & $>35$ & Very steep & 2.00 \\
\hline
\end{tabular}

Soil texture: L: loam, SCL: sandy clay loam, SL: sandy loam, LS: loamy sand, CL: clay loam, SC: sandy clay, SiL: silty loam, SiCL: silty clay loam, Si: silt, C: clay, SiC: silty clay, S: sand.

Table 2. Definition of parent material and rock fragments.

\begin{tabular}{|c|c|c|c|c|c|c|c|}
\hline \multicolumn{4}{|c|}{ Parent material $(\mathrm{M})$} & \multicolumn{4}{|c|}{ Rock fragments (R) } \\
\hline Class & Parent material & Description & Index & Class & $\begin{array}{c}\text { Rock } \\
\text { fragment } \\
(\%)\end{array}$ & $\begin{array}{c}\text { Descripti } \\
\text { on }\end{array}$ & Index \\
\hline M1 & $\begin{array}{l}\text { Lime stone, } \\
\text { dolomite, non- } \\
\text { friable, sand } \\
\text { stone, hard } \\
\text { limestone layer }\end{array}$ & Coherent & 1.00 & $\mathbf{R} 1$ & $>60$ & $\begin{array}{l}\text { Very } \\
\text { stony }\end{array}$ & 1.00 \\
\hline M2 & $\begin{array}{l}\text { Marine limestone, } \\
\text { Friable }\end{array}$ & Moderate & 1.66 & $\mathbf{R 2}$ & $20-60$ & Stony & 1.33 \\
\hline M3 & $\begin{array}{l}\text { Calcareous clay, } \\
\text { clay, sandy } \\
\text { formation, } \\
\text { alluvium, } \\
\text { colluvium }\end{array}$ & $\begin{array}{l}\text { Soft to } \\
\text { friable }\end{array}$ & 2.00 & $\mathbf{R 3}$ & $<20$ & $\begin{array}{l}\text { Slightly } \\
\text { stony }\end{array}$ & 2.00 \\
\hline
\end{tabular}


Table 3. Definition of Soil depth and Drainage.

\begin{tabular}{|c|c|c|c|c|c|c|c|}
\hline \multicolumn{4}{|c|}{ Soil depth $(P)$} & \multicolumn{4}{|c|}{ Drainage (D) } \\
\hline Class & $\begin{array}{c}\text { Soil } \\
\operatorname{depth}(\mathrm{cm})\end{array}$ & Description & Index & Class & Drainage & Description & Index \\
\hline P1 & $>75$ & Deep & 1.00 & D1 & $\begin{array}{c}\text { Well } \\
\text { drained }\end{array}$ & Good & 1.00 \\
\hline $\mathbf{P 2}$ & $30-75$ & Moderate & 1.33 & D2 & $\begin{array}{l}\text { Imperfectly } \\
\text { drained }\end{array}$ & Moderate & 1.33 \\
\hline P3 & $15-30$ & Shallow & 1.66 & D3 & $\begin{array}{c}\text { Poor } \\
\text { drained }\end{array}$ & Poor & 2.00 \\
\hline P4 & $<15$ & $\begin{array}{c}\text { Very } \\
\text { shallow }\end{array}$ & 2.00 & & & & \\
\hline
\end{tabular}

Table 4. Definition of Hydraulic Conductivity and Water holding capacity.

\begin{tabular}{|c|c|c|c|c|c|c|c|}
\hline \multicolumn{4}{|c|}{ Hydraulic Conductivity $(\mathrm{H})$} & \multicolumn{4}{|c|}{ Water holding capacity(W) } \\
\hline Class & $\begin{array}{c}\text { Hydraulic } \\
\text { Conductivity }(\mathrm{cm} / \mathrm{h})\end{array}$ & Description & Index & Class & $\begin{array}{c}\text { Water } \\
\text { holding } \\
\text { capacity } \\
(\%)\end{array}$ & Description & Index \\
\hline H1 & $<0.5$ & Good & 1.00 & W1 & $>50 \%$ & Good & 1.00 \\
\hline $\mathbf{H 2}$ & $0.5-2$ & Moderate & 1.33 & W2 & $50-20 \%$ & Moderate & 1.33 \\
\hline H3 & $2-6.25$ & Poor & 1.66 & W3 & $20-15 \%$ & Poor & 1.66 \\
\hline H4 & $>6.25$ & Very Poor & 2.00 & W4 & $<15 \%$ & Very poor & 2.00 \\
\hline
\end{tabular}

Table 5. Definition of Electrical Conductivity (EC)andSoil Reaction (pH).

\begin{tabular}{cccccccc}
\hline \multicolumn{3}{c}{ Electrical Conductivity $(\mathbf{C})$} & \multicolumn{5}{c}{ Soil Reaction $(\mathbf{O})$} \\
\hline Class & $\begin{array}{c}\text { Electrical } \\
\text { Conductivity } \\
(\mathbf{d S} / \mathbf{m})\end{array}$ & Description & Index & Class & $\begin{array}{c}\text { Soil } \\
\text { Reaction }\end{array}$ & Description & Index \\
\hline C1 & $<4$ & Low & 1.00 & $\mathbf{O 1}$ & $5.5-7$ & Low & 1.00 \\
C2 & $4-8$ & Moderate & 1.33 & $\mathbf{O 2}$ & $7-7.8$ & Moderate & 1.33 \\
C3 & $8-16$ & High & 1.66 & $\mathbf{O 3}$ & $7.78-8.5$ & High & 1.66 \\
C4 & $>16$ & Very high & 2.00 & $\mathbf{O 4}$ & $>8.5$ & Very high & 2.00 \\
\hline
\end{tabular}

Table 6. Definition of Exchangeable Sodium percentage (ESP) and Calcium carbonate $\left(\mathrm{CaCO}_{3}\right)$.

\begin{tabular}{|c|c|c|c|c|c|c|c|}
\hline \multicolumn{4}{|c|}{ Exchangeable Sodium percentage (G) } & \multicolumn{4}{|c|}{ Calcium carbonate $(\mathbf{N})$} \\
\hline Class & $\begin{array}{c}\text { Exchangeable } \\
\text { Sodium } \\
\text { percentage } \\
(\%)\end{array}$ & Description & Index & Class & $\begin{array}{c}\text { Calcium } \\
\text { carbonate } \\
\text { (g/Kg) }\end{array}$ & Description & Index \\
\hline G1 & $<10$ & Low & 1.00 & N1 & $<50$ & Low & 1.00 \\
\hline G2 & $10-15$ & Moderate & 1.33 & $\mathbf{N} 2$ & $50-100$ & Moderate & 1.33 \\
\hline G3 & $15-20$ & High & 1.66 & $\mathbf{N 3}$ & $100-150$ & High & 1.66 \\
\hline G4 & $>20$ & Very high & 2.00 & N4 & $>150$ & Very high & 2.00 \\
\hline
\end{tabular}

\section{Result and Discussion:}

Geomorphologic features and soils.

The geomorphologic units were identified by analyzing the landscape extracted from satellite imagery with the aid of Digital Elevation Model (DEM). The geomorphology map of the investigated area (Figure 3) shows one main landscape is flood plain can be divided into four landforms as follows (table 7):

1) Overflow basins (OB) covered $17.29 \%$ (5838 ha) of the total area. The soils in this landform were classified into VerticTorrifluvents and TypicTorrifluvents.

2) High River terraces (RT1) covered $24.71 \%$ (8344 ha) of the total area. The soils in this landform were classified into VerticTorrifluvents and TypicTorrifluvents.

3) Moderate River terraces (RT2) covered $55.51 \%$ (18739 ha) of total area. The soils in this landform were classified into VerticTorrifluvents.

4) Low River terraces (RT3) covered $2.49 \%$ (839 ha) of total area. The soils in this landform were classified into TypicTorrifluvents. 
Table 7. Landforms and soils classification of the investigated area.

\begin{tabular}{|c|c|c|c|c|c|}
\hline Landform & $\begin{array}{c}\text { Mapping } \\
\text { unit }\end{array}$ & $\begin{array}{l}\text { Profile } \\
\text { No. }\end{array}$ & Soil Classification & $\begin{array}{c}\text { Area } \\
\text { (ha) }\end{array}$ & Area \% \\
\hline Overflow basins & OB & $\begin{array}{l}2,3 \text { and } \\
4\end{array}$ & $\begin{array}{l}\text { Profile 2:VerticTorrifluvents } \\
\text { Profile 3:TypicTorrifluvents. } \\
\text { Profile 4:VerticTorrifluvents. }\end{array}$ & 5838 & 17.29 \\
\hline High River terraces & RT1 & 1 and 9 & $\begin{array}{l}\text { Profile 1:TypicTorrifluvents. } \\
\text { Profile 9:VerticTorrifluvents. }\end{array}$ & 8344 & 24.71 \\
\hline $\begin{array}{l}\text { Moderate River } \\
\text { terraces }\end{array}$ & RT2 & $\begin{array}{l}5,6 \text { and } \\
8\end{array}$ & VerticTorrifluvents. & 18739 & 55.51 \\
\hline Low River terraces & RT3 & 7 & TypicTorrifluvents. & 839 & 2.49 \\
\hline Total area (ha) & & & & 33760 & 100.00 \\
\hline
\end{tabular}

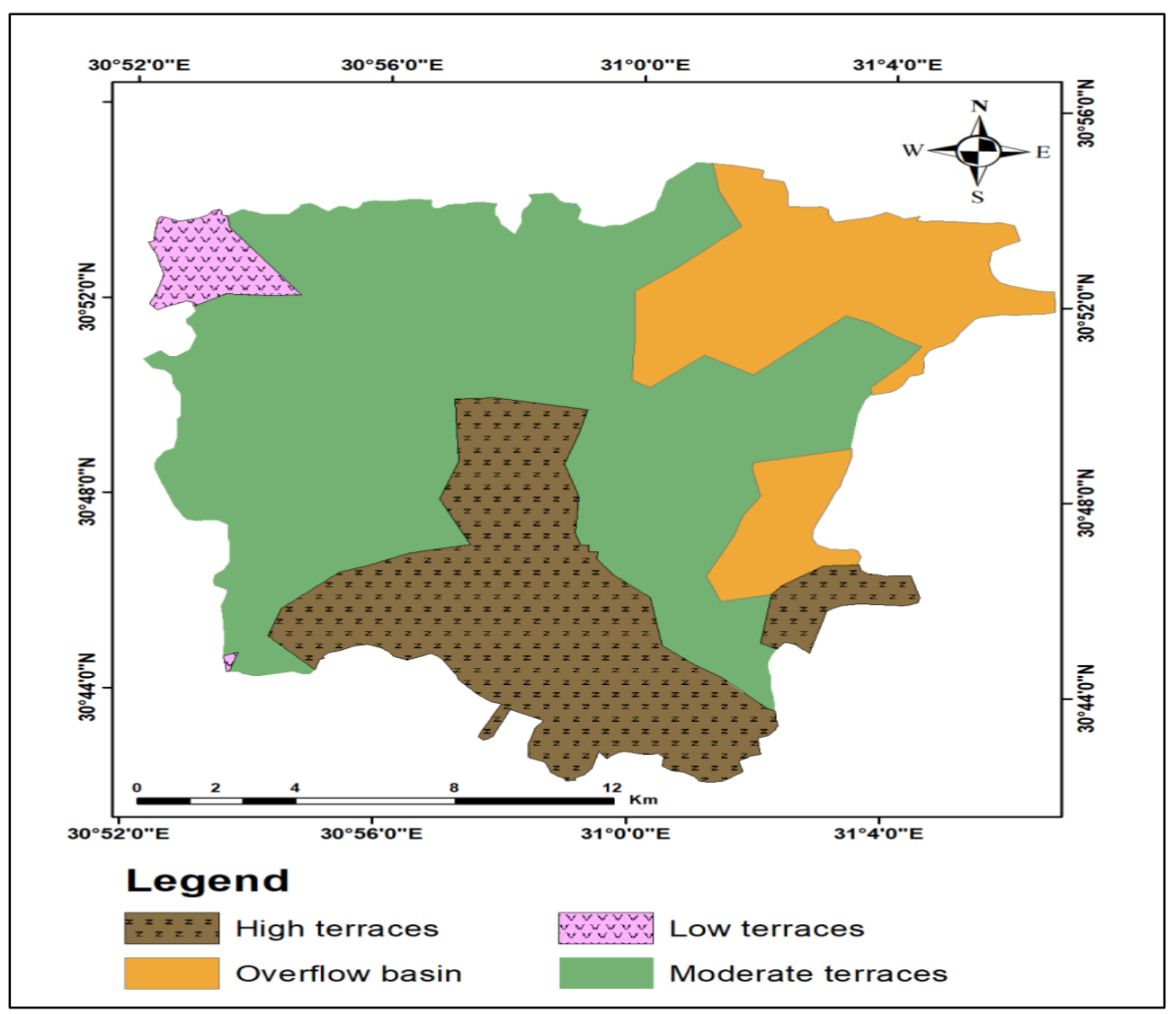

Fig. 3: Geomorphologic map of Tanta area.

Soil Quality Index (SQI) and rating system.

Twelve layers were used to assess Soil Quality Index (SQI) in the studied area, including physical and chemical properties. These layers were created in a geographic information system (GIS) using the spatial analyst tool. The Landsat $8 \mathrm{ETM}^{+}$ image of the studied area and the digital elevation model were used to establish the main land type layer, this layer was used as a base map in the geographic information system. The SQI model established by ElNagar et al., (2013).Soil is an essential factor in evaluating the quality of an ecosystem, especially in the arid and semi-arid zones. Soil physical and chemical properties related to soil quality include water storage and retention capacity and resistance to erosion. The physical soil quality index (PSQI) was evaluated depending upon drainage condition, rock fragments $(\%)$ slope gradient $(\%)$, soil texture class, soil depth $(\mathrm{cm})$, parent material, hydraulic conductivity $(\mathrm{cm} / \mathrm{h})(\mathrm{H})$ and water holding capacity $(\%)(\mathrm{W})$.The chemical soil quality index (CSQI) was evaluated depending upon electrical conductivity $(\mathrm{dS} / \mathrm{m})(\mathrm{C})$, soil reaction $(\mathrm{O})$, exchangeable sodium percentage (cmolc/kg soil) $(\mathrm{G})$ and calcium carbonate $(\mathrm{g} / \mathrm{kg})(\mathrm{N})$. The mathematical formula expressing Quality is as follows:

Soil Quality Index $(\mathrm{SQI})=(\mathrm{T} \times \mathrm{D} \times \mathrm{P} \times \mathrm{M} \times \mathrm{S} \times \mathrm{R}$ $\times \mathrm{H} \times \mathrm{W} \times \mathrm{C} \times \mathrm{O} \times \mathrm{G} \times \mathrm{N})^{1 / 8}$ 
Each factor is rated on a scale from 1 to 2 and the resultant index, lies between 1.13 and 1.45 , and is set against a scale placing the soil in one of the following four Quality classes (Table 8):

Table 8. Soil quality classes and rating.

Soil Quality Index

\begin{tabular}{c} 
Grade \\
\hline I \\
II \\
III
\end{tabular}

Soil quality Index Model and rating system.

In this model, interpretation criteria are modeled based on soil physical and chemical properties traditionally incorporate (El-Nagar et al., 2013). The structure organization of the El-Nagar model is summarized in Figure 4.

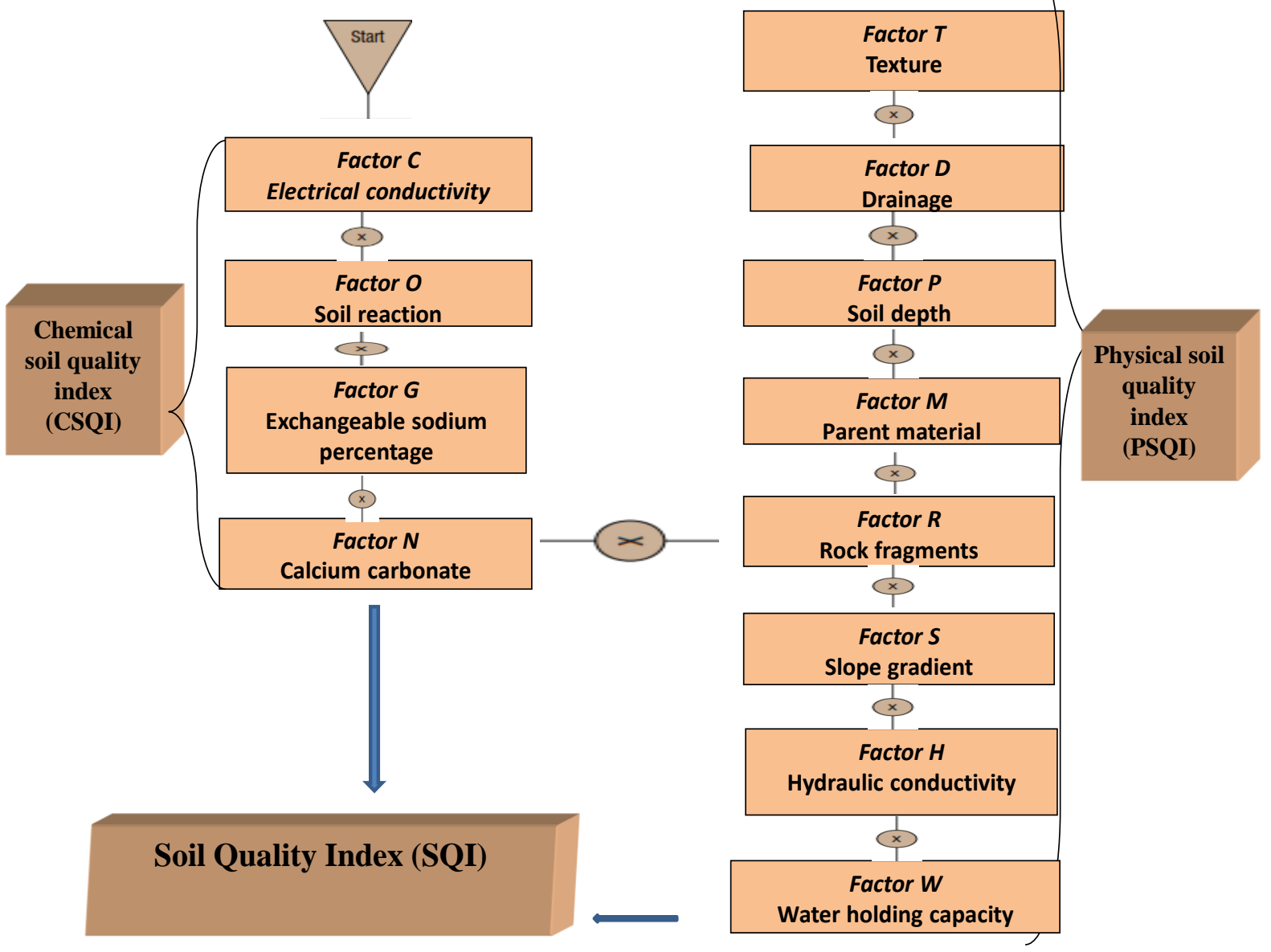

Fig. 4: Model of Soil Quality Index.

Assessment of Physical Soil Quality Index (PSQI).

The results indicate that the areas of moderate physical quality index (value $=1.13-1.45$ ) represents $100 \%$ of the total area (i.e.33760 ha). Table 9 to12 illustrates the general characteristics, classes and scores of the soil physical quality index and their map is shown in figure 5 using GIS. 
Table 9. Values of the Physical factors of Soil Quality of the studied soils of the investigated area.

\begin{tabular}{ccccccccc}
\hline $\begin{array}{c}\text { Mapping } \\
\text { unit }\end{array}$ & Texture & Drainage & $\begin{array}{c}\text { Effective } \\
\text { depth } \\
\text { (cm) }\end{array}$ & $\begin{array}{c}\text { Parent } \\
\text { materials }\end{array}$ & $\begin{array}{c}\text { Rock } \\
\text { Fragments }\end{array}$ & $\begin{array}{c}\text { Slop } \\
\text { Gradient }\end{array}$ & $\begin{array}{c}\text { Hydraulic } \\
\text { conductivity } \\
\text { (cm/h) }\end{array}$ & $\begin{array}{c}\text { Water } \\
\text { holding } \\
\text { capacity (\%) }\end{array}$ \\
\hline RT1 & $\begin{array}{c}\text { Clay } \\
\text { loam }\end{array}$ & $\begin{array}{c}\text { Moderate } \\
\text { drained }\end{array}$ & 80 & Alluvium & No stones & Flat & $3.13 \times 10^{-3}$ & 43.9 \\
& $\begin{array}{c}\text { Silty } \\
\text { clay }\end{array}$ & $\begin{array}{c}\text { Moderate } \\
\text { drained }\end{array}$ & 86.6 & Alluvium & No stones & Flat & $2.13 \times 10^{-3}$ & 47.6 \\
RT2 & $\begin{array}{c}\text { loam } \\
\text { Clay }\end{array}$ & $\begin{array}{c}\text { Good } \\
\text { drained } \\
\text { loam }\end{array}$ & 100 & Alluvium & No stones & Flat & $1.59 \times 10^{-3}$ & 41.3 \\
OB & $\begin{array}{c}\text { Clay } \\
\text { loam }\end{array}$ & $\begin{array}{c}\text { Good } \\
\text { drained }\end{array}$ & 100 & Alluvium & No stones & Flat & $5.28 \times 10^{-3}$ & 44.6 \\
\hline
\end{tabular}

Table 10. Soil physical characteristics of the investigated area.

\begin{tabular}{ccccccccc}
\hline $\begin{array}{c}\text { Mapping } \\
\text { unit }\end{array}$ & $\begin{array}{c}\text { Texture } \\
\text { (T) }\end{array}$ & $\begin{array}{c}\text { Drainage } \\
\text { (D) }\end{array}$ & $\begin{array}{c}\text { Effective } \\
\text { depth (P) }\end{array}$ & $\begin{array}{c}\text { Parent } \\
\text { materials } \\
\text { (M) }\end{array}$ & $\begin{array}{c}\text { Rock } \\
\text { Fragments } \\
\text { (R) }\end{array}$ & $\begin{array}{c}\text { Slop } \\
\text { Gradient } \\
\text { (S) }\end{array}$ & $\begin{array}{c}\text { Hydraulic } \\
\text { conductivity } \\
\text { ( H) }\end{array}$ & $\begin{array}{c}\text { Water } \\
\text { holding } \\
\text { capacity } \\
\text { (W) }\end{array}$ \\
\hline RT1 & T1 & D2 & P1 & M3 & R3 & S1 & H1 & W2 \\
RT2 & T2 & D2 & P1 & M3 & R3 & S1 & H1 & W2 \\
RT3 & T1 & D1 & P1 & M3 & R3 & S1 & H1 & W2 \\
OB & T1 & D1 & P1 & M3 & R3 & S1 & H1 & W2 \\
\hline
\end{tabular}

Table 11. Assessment of physical Soil Quality Index of the study area.

\begin{tabular}{|c|c|c|c|c|c|c|c|c|c|c|}
\hline $\begin{array}{c}\text { Mappi } \\
\text { ng } \\
\text { unit }\end{array}$ & $\begin{array}{l}\text { Textu } \\
\text { re } \\
(\mathbf{T})\end{array}$ & $\begin{array}{l}\text { Draina } \\
\text { ge (D) }\end{array}$ & $\begin{array}{l}\text { Effecti } \\
\text { ve } \\
\text { depth } \\
(P)\end{array}$ & $\begin{array}{l}\text { Parent } \\
\text { materi } \\
\text { als }(\mathbf{M})\end{array}$ & $\begin{array}{c}\text { Rock } \\
\text { Fragme } \\
\text { nts }(\mathbf{R})\end{array}$ & $\begin{array}{c}\text { Slop } \\
\text { Gradie } \\
\text { nt }(\mathbf{S})\end{array}$ & $\begin{array}{c}\text { Hydrauli } \\
\text { c } \\
\text { conducti } \\
\text { vity }(H)\end{array}$ & $\begin{array}{l}\text { Water } \\
\text { holdin } \\
\text { g } \\
\text { capaci } \\
\text { ty }(W)\end{array}$ & $\begin{array}{c}\text { Physi } \\
\text { cal } \\
\text { soil } \\
\text { qualit } \\
\text { y } \\
\text { index }\end{array}$ & $\begin{array}{c}\text { Gra } \\
\text { de }\end{array}$ \\
\hline RT1 & 1.00 & 1.33 & 1.00 & 2.00 & 2.00 & 1.00 & 1.00 & 1.33 & 1.27 & II \\
\hline RT2 & 1.33 & 1.33 & 1.00 & 2.00 & 2.00 & 1.00 & 1.00 & 1.33 & 1.32 & II \\
\hline RT3 & 1.00 & 1.00 & 1.00 & 2.00 & 2.00 & 1.00 & 1.00 & 1.33 & 1.23 & II \\
\hline OB & 1.00 & 1.00 & 1.00 & 2.00 & 2.00 & 1.00 & 1.00 & 1.33 & 1.23 & II \\
\hline
\end{tabular}

Table 12.Distribution of Physical Soil Quality Index (PSQI) of the study area

\begin{tabular}{cccccc}
\hline $\begin{array}{c}\text { Physical Soil Quality Index } \\
\text { (PSQI) }\end{array}$ & Grade & Class & Mapping unit & Area (ha) & Area \% \\
\hline$<1.13$ & I & High quality & - & - & - \\
$1.13-1.45$ & II & Moderate quality & RT1, RT2, RT3 and & 33760 & 100 \\
$>1.45$ & III & Low quality & - & - & - \\
\hline
\end{tabular}




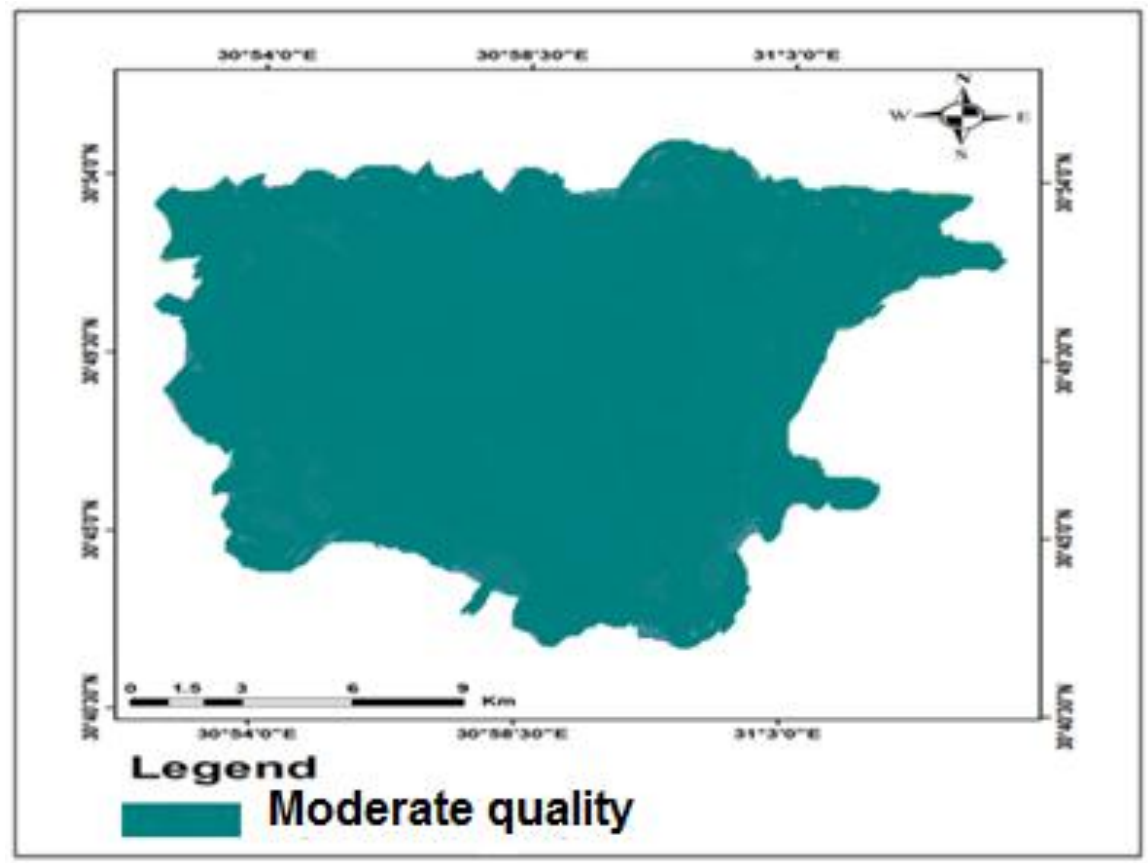

Fig. 5: Map of physical soil quality

Assessment of Chemical Soil Quality Index (CSQI).

The results indicate that the areas of high soil quality index (value <1.13) represent $19.78 \%$ of the total area (i.e. $6677 \mathrm{ha})$ and the areas of moderate quality index $($ value $=1.13-1.45)$ represents $80.22 \%$ of the total area (i.e.27083 ha). Table 13 to 16 illustrates the general characteristics, classes and scores of the soil chemical quality index and their map is shown in figure 6 using GIS.

Table 13.Values of the chemical factors of Soil Quality of the studied soils of the investigated area.

\begin{tabular}{ccccc}
\hline Mapping unit & $\begin{array}{c}\text { Electrical } \\
\text { conductivity (C) }\end{array}$ & Soil reaction (O) & $\begin{array}{c}\text { Exchangeable } \\
\text { sodium } \\
\text { percentage (G) }\end{array}$ & Calcium carbonate (N) \\
\hline RT1 & 1.79 & 7.70 & 25.31 & 26.36 \\
RT2 & 0.79 & 7.83 & 19.22 & 22.42 \\
RT3 & 0.71 & 7.76 & 13.36 & 11.81 \\
OB & 0.86 & 7.65 & 16.09 & 33.60 \\
\hline
\end{tabular}

Table 14. Soil chemical characteristics of the investigated area.

\begin{tabular}{lcccc}
\hline Mapping unit & $\begin{array}{c}\text { Electrical } \\
\text { conductivity }(\mathbf{C})\end{array}$ & $\begin{array}{c}\text { Soil reaction } \\
(\mathbf{O})\end{array}$ & $\begin{array}{c}\text { Exchangeable sodium } \\
\text { percentage }(\mathbf{G})\end{array}$ & $\begin{array}{c}\text { calcium carbonate } \\
(\mathbf{N})\end{array}$ \\
\hline RT1 & $\mathrm{C} 1$ & $\mathrm{O} 2$ & $\mathrm{G} 4$ & $\mathrm{~N} 1$ \\
RT2 & $\mathrm{C} 1$ & $\mathrm{O} 3$ & $\mathrm{G} 3$ & $\mathrm{~N} 1$ \\
RT3 & $\mathrm{C} 1$ & $\mathrm{O} 2$ & $\mathrm{G} 2$ & $\mathrm{~N} 1$ \\
OB & $\mathrm{C} 1$ & $\mathrm{O} 2$ & $\mathrm{G} 3$ & $\mathrm{~N} 1$ \\
\hline
\end{tabular}

Table 15. Assessment of chemical Soil Quality Index of the study area.

\begin{tabular}{lcccccc}
\hline $\begin{array}{l}\text { Mapping } \\
\text { unit }\end{array}$ & $\begin{array}{c}\text { Electrical } \\
\text { conductivity } \\
\text { (C) }\end{array}$ & $\begin{array}{c}\text { Soil } \\
\text { reaction } \\
(\mathbf{O})\end{array}$ & $\begin{array}{c}\text { Exchangeable } \\
\text { sodium } \\
\text { percentage } \\
(\mathbf{G})\end{array}$ & $\begin{array}{c}\text { calcium } \\
\text { carbonate } \\
(\mathbf{N})\end{array}$ & $\begin{array}{c}\text { Chemical Soil } \\
\text { quality index }\end{array}$ & Grade \\
\hline RT1 & 1.00 & 1.33 & 2.00 & 1.00 & 1.13 & II \\
RT2 & 1.00 & 1.66 & 1.66 & 1.00 & 1.13 & II \\
RT3 & 1.00 & 1.33 & 1.33 & 1.00 & 1.07 & I \\
OB & 1.00 & 1.33 & 1.66 & 1.00 & 1.10 & I \\
\hline
\end{tabular}


Table 16. Distribution of Chemical Soil Quality Index (PSQI) of the study area

\begin{tabular}{cccccc}
\hline $\begin{array}{c}\text { Chemical Soil Quality Index } \\
\text { (PSQI) }\end{array}$ & $\begin{array}{c}\text { Grade } \\
\text { Distribution }\end{array}$ & Class & Mapping unit & Area (ha) Area \% \\
\hline$<1.13$ & I & High quality & RT3 and OB & 6677 & 19.78 \\
$1.13-1.45$ & II & Moderate quality & RT1 and RT2 & 27083 & 80.22 \\
$>1.45$ & III & Low quality & - & - & - \\
\hline
\end{tabular}

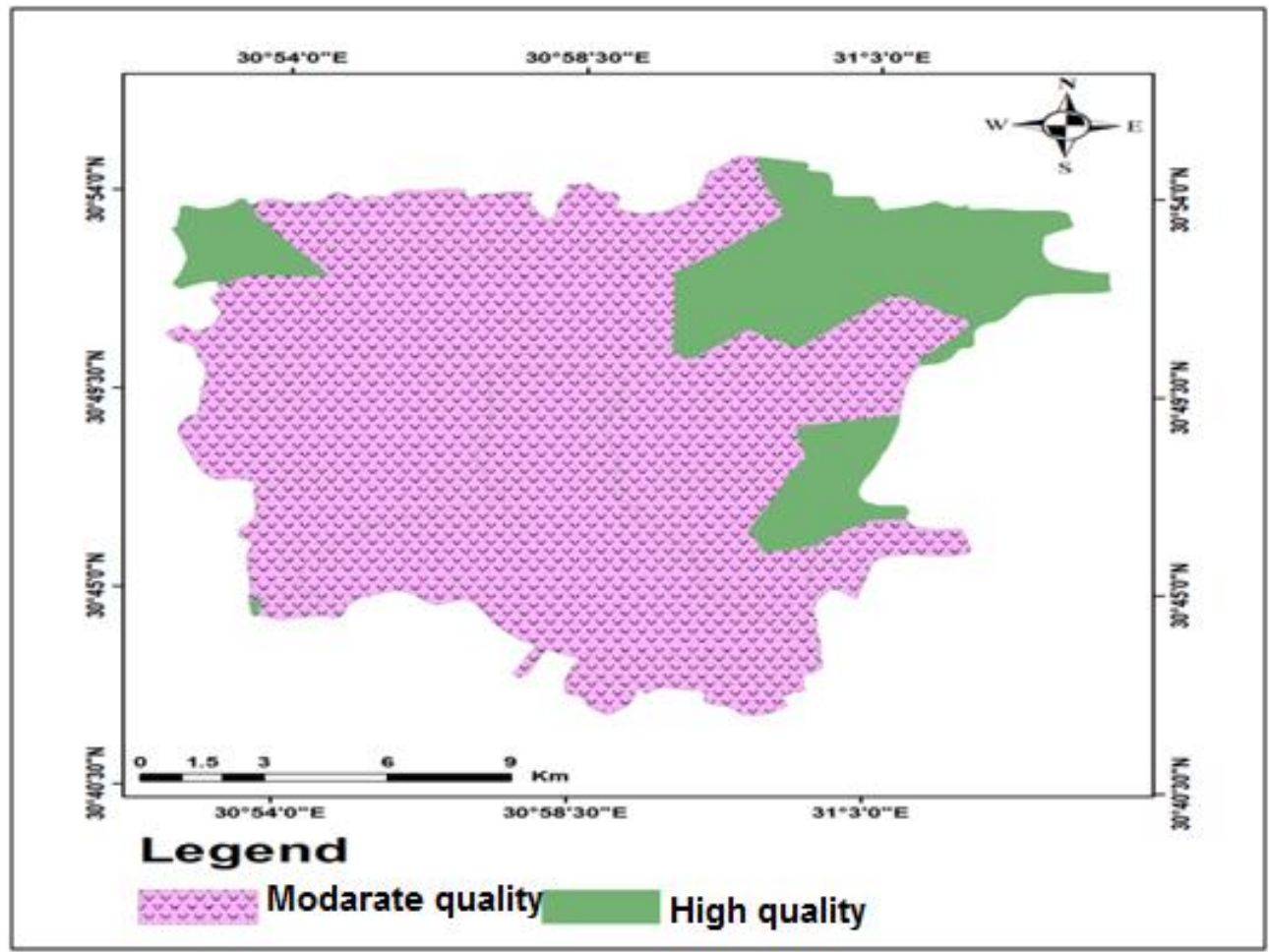

Fig. 6: Map of chemical soil quality

Assessment of Soil Quality Index (SQI).

The results indicate that the areas of moderate quality index (value $=1.13-1.45$ ) represents $44.49 \%$ of the total area (i.e.15021 ha) in RT1, RT3 and OB mapping units and the areas of low soil quality index (value >1.45) represents $55.51 \%$ of the total area (i.e.18739ha) in RT2 mapping unit. The low soil quality dominates the areas characterized by shallow depth, poor drainage and Hydraulic conductivity. Table 16 illustrates the assessment of Soil quality index of the study area and the map of soil quality is shown in figure 7.

Table 16. Assessment of Soil Quality Index of the study area.

\begin{tabular}{ccccc}
\hline Mapping unit & $\begin{array}{c}\text { Physical soil } \\
\text { quality index }\end{array}$ & $\begin{array}{c}\text { Chemical Soil } \\
\text { quality index }\end{array}$ & $\begin{array}{c}\text { Soil quality } \\
\text { index }\end{array}$ & Grade \\
\hline RT1 & 1.27 & 1.13 & 1.43 & II \\
RT2 & 1.32 & 1.13 & 1.49 & III \\
RT3 & 1.23 & 1.07 & 1.31 & II \\
OB & 1.23 & 1.10 & 1.35 & II \\
\hline
\end{tabular}

Table 17. Distribution of Soil Quality Index (SQI) of the study area.

\begin{tabular}{cccccc}
\hline Soil Quality Index (SQI) & $\begin{array}{c}\text { Grade } \\
\text { Distribution }\end{array}$ & Class & Mapping unit & Area (ha) & Area \% \\
& I & High quality & & & \\
$1.13-1.45$ & II & Moderate quality & RT1, RT3 and OB & 15021 & 44.49 \\
$>1.45$ & III & Low quality & RT2 & 18739 & 55.51 \\
\hline
\end{tabular}




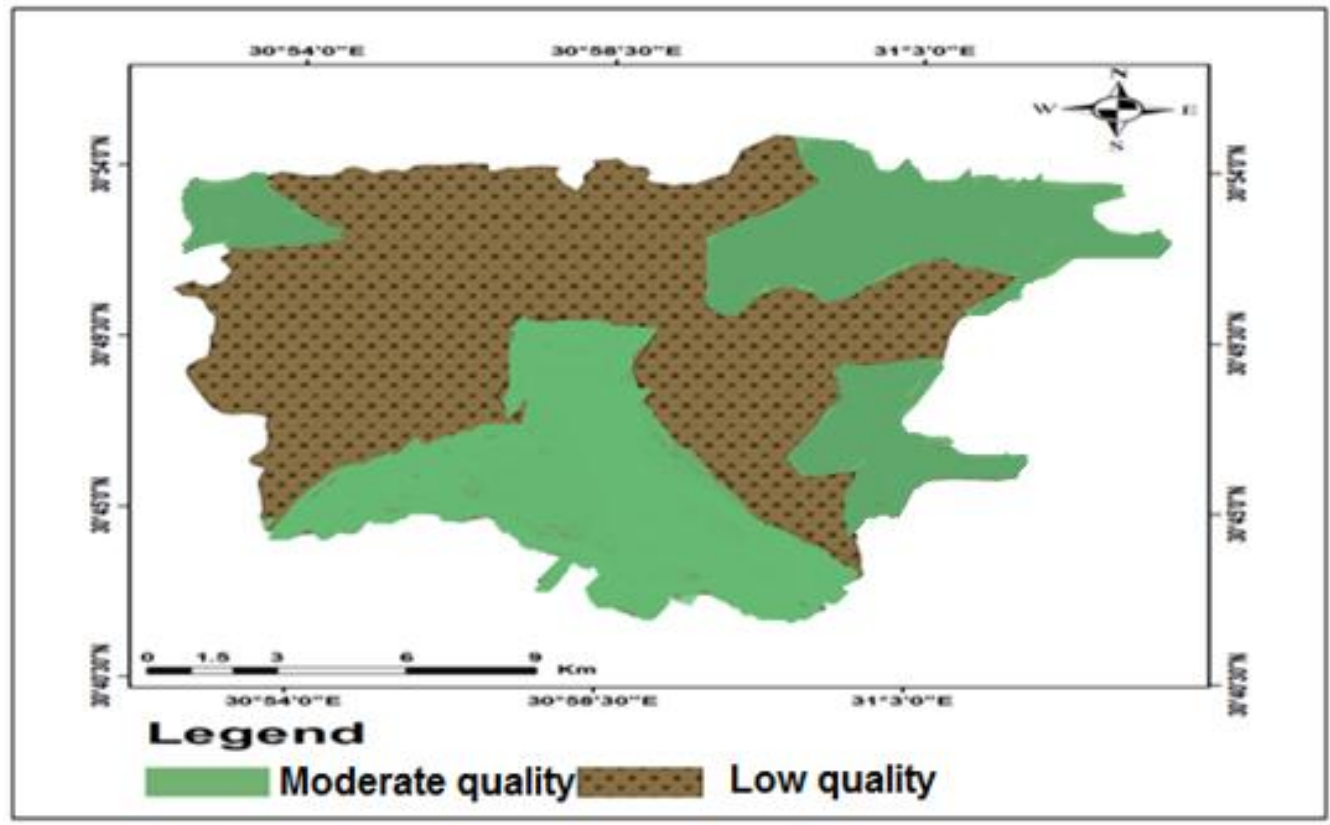

Fig.7: Map of Soil Quality Index.

\section{Conclusion}

It could be concluded that the soil quality index (SQI) model could provide a valuable quantitative assessment of twelve soil characteristics with important information that could help in protecting and sustaining natural resources. In this model soil quality was evaluated based on two important soil quality indices (physical and chemical) that have great impact on that phenomenon. Remote sensing and GIS techniques are very helpful to evaluate soil quality index and produce a physiographic map of soil quality index.

\section{Reference}

Abdel Rahman, M.A.E. and Tahoun, S. 2019. GIS model-builder based on comprehensive geostatistical approach to assess soil quality. Remote Sensing Applications: Society and Environment, 13: 204-214.

Abu-hashim, M.; Mohamed, E. and Belalb, A. 2015. Identification of potential soil water retention using hydric numerical model at arid regions by land-use changes. International Soil and Water Conservation Research, 3: 305-315.

Adeyolanu, O. D.; Are, K. S.; Oluwatosin, G. A.; Ayoola, O. T. and Adelana, A. O. 2013. Evaluation of two methods of soil quality assessment as influenced by slash and burn in tropical rainforest ecology of Nigeria. Archives of Agronomy and Soil Science, 59(12): 1725-1742.

Afify, A.A.; Arafat, S.M.; AboelGhar, M. and Afify, M. N. 2011. Delineating rice belt cultivation in the Nile pro-delta of Vertisols using remote sensing data of Egypt Sat-1. J. Agric. Res., 35 (6): 2263-2279.
Afify, A.A.; Arafat, S.M.; AboelGhar, M.; Afify, N. M. and Ahmed, I. F. 2008. Retreating rate estimation of the fertile alluvium in Nile Delta under the urban encroachment, using remote sensing data and GIS techniques. In: 16th International Symposium on Remote Sensing and Spatial Information, General Organization of Remote Sensing Syrian Arab Republic Damascus - Syrian Arab Republic, 10-12 November

Allen, D.E.; Singh, B.P. and Dalal, R.C. 2011.Soil Health Indicators, Soil Health and Climate Change: A Review of Current Knowledge. In B.P. Singh et al. (eds.), Soil Health and Climate Change, Soil Biol., 29: 25-45.

Andrews, S. S.; Karlen, D. L. and Cambardella, C. A. 2004. The soil management assessment framework. Soil Science Society of America Journal, 68(6): 1945-1962.

Anon. 1975. Munsell color charts Macbeth Division of kollmorgen Corporation, 2441 North Cavert Street Batimore, Maryland, USA.

Arshad, M. A. and Martin, S. 2002. Identifying critical limits for soil quality indicators in agroecosystems. Agriculture, Ecosystems and Environment 88(2): 153-160.

Belal, A. A. and Moghanm, F.S. 2011. Detecting urban growth using remote sensing and GIS techniques in Al Gharbiya governorate, Egypt. The Egyptian Journal of Remote Sensing and Space Sciences, 14(2):73-79.

Blum, W. E. 2003.European soil protection strategy. Journal of Soils and Sediments, 3(4): 242-242.

Cambardella, C.; Moorman, T.; Andrews, S. and Karlen, D. 2004. Watershed-scale assessment of soil quality in the loess hills of South west Iowa. Soil and Tillage Research, 78(2): 237-247. 
Craig, A. and Arlene, J. 2002. Soil quality field tools: experiences of USDA-NRCS soil quality institilte. Agronomy Journal, 94: 33-38.

Daneshvar, M. R. M.; Khatami, F. and Shirvani, S. 2017. GIS-based land suitability evaluation for building height construction using an analytical process in the Mashhad city, NE Iran. Model. Earth Syst. Environ., 3:16.

De la Rose, D. 2005. Soil Quality Evaluation and Monitoring Based on Land Evaluation. Land Degradation Development, 16(6): 551-559.

Doran, J. W. 2002. Soil health and global sustainability: translating science into practice, Agr. Ecosyst. Environ, 88 (2): 119-127.

Doran, J.W. and Jones, A. J. 1996. Methods for assessing soil quality: Soil Science Society of America Inc.

Doran, J.W. and Parkin, T.B. 1994. Defining and assessing soil quality. In J.W. Doran, D.C. Coleman, D.F. Bezdicek, and B.A. Stewart (ed.) Defining Soil Quality for a Sustainable Environment. Soil Sci. Soc. Amer., Madison, Wisconsin, 1-21.

El Baroudy, A. A. 2015.Assessing Long Term Changes of Productivity in Some Floodplain Soils, Egypt, Using Spatial Analyses Techniques. Egypt. J. Soil Sci., 55(2):155-170.

EL-Asmar, H. and Hereher, M. 2011. Change detection of the coastal zone east of the Nile Delta using remote sensing. Environmental Earth Sciences, 62:769-777.

Elnaggar, A. A.; El-Hamdi, Kh. H. A.; Belal, B.A.; El-Kafrawy, M. M. 2013. Soil classification of Bahariya Oasis using remote sensing and GIS techniques. J. Soil Sci. and Agric. Eng., Mansoura Univ., 4(9): 921-947.

Embabi, N.S. 2018. Landscapes and landforms of Egypt: landforms and evolution. In: Migoń,P. and Wroclaw, P. (eds.) World geomorphological landscapes. Springer International Publishing AG, 3-336.

Estefan, G.; Sommer, R. and Ryan, J. 2013. Method of soil, plant and water Analysis: A manual for the west Asia and North Africa region. International Center for Agricultural Research in the Dry Areas, $243 p$.

FAO. 2006. Guidelines for soil description. $4^{\text {th }} E d$., Food and Agriculture Organization Food and Agriculture Organization (FAO), Rome, Italy.

Govers, G.; Merckx, R.; van Oost, K. and van Wesemael, B. 2013. Managing soil organic carbon for global benefits: a STAP technical report. Global Environmental Facility, Washington, DC, 1-72.

Hossain, M. S. and Das, N. G. 2010. GIS-based multi-criteria evaluation to land suitability modeling for giant prawn (Macrobrachiumrosenbergii) farming in Companig on jUpazila of Noakhali, Bangladesh. Computers Electron Agric., 70(1):172-186.
Ismael, H. 2015. The Effectiveness of Using MODIS Products for Monitoring Climate Change Risks over the Nile Delta, Egypt. International Journal of Environmental Monitoring and Analysis, 3(6): 382-396.

Joerin, F.; Theriault, M. and Musy, A. 2001. Using GIS and outranking multi-criteria analysis for land use suitability assessment. Int. J. Geogr. Inf. Sci., 10(8):321-339.

Karlen, D.; Andrews, S. S.; Wienhold, B. J. and Zobeck, T. M. 2008. Soil quality assessment: past, present and future. Electronic Journal of Integrative Biosciences, 6(1): 3-14.

Karlen, D.; Mausbach, M.; Doran, J.; Cline, R.; Harris, R. and Schuman, G. 1997. Soil quality: a concept, definition, and framework for evaluation (a guest editorial). Soil Science Society of America Journal, 61(1): 4-10.

Karlen, D.; Stott, D.; Cambardella, C.; Kremer, R.; King, K. and McCarty, G. 2014. Surface soil quality in five Midwestern cropland conservation effects assessment project watersheds. Journal of Soil and Water Conservation, 69(5):393-401.

Klingebiel, A. and Montgomery, P. 1961. Landcapability classification. In Soil conservation service, Agricultural hand book. No. 210. Washington DC: US Department of Agriculture.

Larson, W. E. and Pierce, F. J. 1994. The dynamics of soil quality as a measure of sustainable management. In: Doran, J. W. (Ed.) Defining Soil Quality for a Sustainable Environment. Soil Science Society of America Special Publication No.35. Madison: Soil Science Society of America and American Society of Agronomy, 37-51.

Liebig, M.; Tanaka, D. and Wienhold, B. J. 2004. Tillage and cropping effects on soil quality indicators in the northern Great Plains. Soil and Tillage Research, 78(2):131-141.

Liniger, H.; Studer, R.M.; Hauert, C. and Gurtner, M. 2011. Sustainable land management in practice. Guidelines and best practices for SubSaharan Africa. TerrAfrica, World Overview of Conservation Approaches and Technologies (WOCAT) and Food and Agricultural Organization of the United Nations (FAO), Rome, Italy, 1-246.

Liu, E.; Yan, C.; Mei, X.; He, W.; Bing, S.; Ding, L.; Liu, Q.; Liu, S. and Fan, T. 2010. Long-term effect of chemical fertilizer, straw and manure on soil chemical and biological properties in northwest China. Geoderma, 158(3-4): 173-180.

Mackay, A. D.; Dominati, E.J.; Rendel, J.M. and Maseyk, F.J.F. 2018. Looking to the future of land evaluation at farm scale.New Zealand Journal of Agricultural Research, 61(3):327-332.

Masoud, A. A. 2016. Geotechnical site suitability mapping for urban land management in Tanta district, Egypt. Arab J. Geosci., 9:340.

Masoud, A. A.; Koike, K.; Mashaly, H.A. and Gergis, F. 2016. Spatio-temporal trends and 
change factors of groundwater quality in an arid area with peat rich aquifers: Emergence of water environmental problems in Tanta District, Egypt. Journal of Arid Environments, 124: 360-376.

Mohamed, E.S.; Belal, A. and Shalaby, A. 2015. Impacts of Soil Sealing on Potential Agriculture in Egypt Using Remote Sensing and GIS Techniques. EURASIAN SOIL SCIENCE, 48 (10):1159-1169.

Moustaf, M.M. 2000. A geostatistical approach to optimize the determination of saturated hydraulic conductivity for large-scale subsurface drainage design in Egypt. Agric. Water Manage., 42(3):291-312.

Nael, M.; Khademi, H. and Hajabbasi, M. 2004. Response of soil quality indicators and their spatial variability to land degradation in Central Iran. Applied Soil Ecology, 27(3): 221232.

Nakajima, T.; Lal, R. and Jiang, S. 2015. Soil quality index of acrosby silt loam in central Ohio. Soil and Tillage Research, 146: 323-328.

Negm, A.M. 2017.The Nile Delta. The handbook of environmental chemistry series, vol. 55. Springer International Publishing AG.

Negm, A.M.; Saavedra, O. and El-Adawy, A. 2017. Nile Delta biography: challenges and opportunities, 55, 3-18, In: Negm, A.M (ed.), The Nile Delta, Barceló, D. and Kostianoy, A.G. (Series eds.), The Handbook of environmental chemistry.

Niekerk, A. v. 2010.A comparison of land unit delineation techniques for land evaluation in the Western Cape, South Africa. Land use policy, 27(3):937-945.

Novak, P.; Vopravil, J. and Lagova, J. 2010. Assessment of the soil quality as a complex of productive and environmental soil function potentials. Soil and Water Res., 5 (3): 113-119.

Omran, E. E. 2017. Land and Groundwater Resources in the Egypt's Nile Valley, Delta, and Its Fringes. Part of the Handbook of Environmental Chemistry book, 73: 45-103.

Ozgoz, E.; Gunal, H.; Acir, N.; Gokmen, F.; Birol, M. and Budak, M. 2013. Soil quality and spatial variability assessment of land use effects in a typichaplustoll. Land Degradation and Development, 24(3): 277-286.

Palm, C.; Sanchez, P.;Ahamed, C. and Awiti, A. 2007. Soils: a contemporary perspective. Annual Review of Environment and Resources, 32:99129.

Rashidi, M.; Seilsepour, M.; Ranjbar, I.; Gholami, M. and Abbassi, S. 2010. Evaluation of some soil quality indicators in the Varamin region, Iran. World Applied Sciences Journal, 9(1):101-108.

Sacco, D.; Moretti, B.; Monaco, S. and Grignani, C. 2015. Six year transition from conventional to organic farming: effects on crop production and soil quality. European Journal of Agronomy, 69: 10-20.
Said, R.1981. The geology and evolution of the River Nile: Springer, New York, N.Y., 151 p.

Schjonning, P.; Elmholt, S. and Christensen, B. T. 2004. Managing soil quality: challenges in modern agriculture. CABI Publishing, Walling ford, 315333.

Sestini, G. 1992. Implications of climatic changes for the Nile Delta. In Climatic Change and the Mediterranean, L. Jeftic, D. Milliman, and G. Sestini (Eds.), 535-601.

Seybold, C.; Mausbach, M.; Karlen, D. and Rogers, H. 1997.Quantification of soil quality. Soil processes and the carbon cycle, 387-404.

Shirani, H.; Hajabbasi, M.; Afyuni, M. and Hemmat, A. 2002.Effects of farm yard manure and tillage systems on soil physical properties and corn yield in central Iran. Soil and Tillage Research, 68(2): 101-108.

Shokr, M. S.; El Baroudya, A.A.; Fullenb, M.A.; El beshbeshy, T.R.; Ramadan, A.R.; Abd El Halim, A.A.; Guerrad, A.J.T. and Jorge, M.C.O. 2016. Spatial distribution of heavy metals in the middle Nile delta of Egypt. International Soil and Water Conservation Research, 4(4): 293303.

Shukla, M. K.; Lal, R. and Ebinger, M. 2006. Determining Soil Quality Indicators by Factor Analysis.Soil and Tillage Research, 87(2): 194204.

Southorn, N. and Cattle, S. 2004. The dynamics of soil quality in livestock grazing system.3rd Australian New Zealand Soils Conference, 5 - 9 December 2004, University of Sydney, Australia.1-7.

USDA. 2014. Keys to soil taxonomy. United State Department of Agriculture (USDA), Natural Resources Conservation Service (NRCS).

Wander, M. M.; Walter,G. L.;Nissen, T. M.; Bollero, G. A. and Andrews, S. S. 2002. Soil quality: Science and Progress Agronomy Journal, 94(1): 23-32.

Wang, X. and Gong, Z. 1998.Assessment and analysis of soil quality changes after eleven years of reclamation in subtropical china.Geoderma, 81 (3-4):339-355.

Wienhold, B. J.; Andrews, S. S. and Karlen, D. L. 2004. Soil quality: A review of the science and experiences in the USA. Environ. Geochem. Health, 26:89-95.

Yang, C.; Yang, L.; Yang, Y. and Ouyang, Z. 2004. Maize root growth and nutrient uptake as influenced by organic manure in continuously and alternately flooded paddy soils. Agricultural Water Management, 70(1): 67-81.

Yao, R.-J.; Yang, J.-S.; Zhang, T.-J.; Gao, P.; Yu, S.P. and Wang, X.-P. 2013. Short-term effect of cultivation and crop rotation systems on soil quality indicators in a coastal newly reclaimed farming area. Journal of Soils and Sediments, 13(8): 1335-1350. 
Younes, M. A. 2015. Natural Gas Geochemistry in the Offshore Nile Delta, Egypt, Advances in Petrochemicals, Vivek Patel, IntechOpen, 27-40.

Zahran, M. A. and Willis, A.J. 2009. The vegetation of Egypt. In: Werger, M.J.A. (ed.) Plant and vegetation, Springer, Berlin, Heidelberg, 2: 305318 .
Zornoza, R.; Acosta, J. A.; Bastida, F.; Domnguez, S. G.; Toledo, D. M. and Faz, A. 2015. Identification of sensitive indicators to assess the interrelationship between soil quality, management practices and human health. SOIL (An interactive open-access journal of the European Geosciences union), 1(1): 173-185.

\section{الخصائص الطبيعية والكيميائية كموئر لجودة التربة فى بعض مناطق طنطا، دلتا التيل، مصر باستخدام تقنيات الاستشعار من بعد ونظم المعلومات الجغرافية. \\ مها على محمد عبدالرازق - هبة شوقى راثد - محمد على عبدالسلام - محمد حسن حمزة عباس قسم الاراضى والمياه- كلية الزراعة- مشتهر - جامعة بنها- مصر . مصند}

تهدف الدراسة الحالية الى تقييم جودة التربة الفيزيائية والكيميائية فى منطقة طنطا بمحافظة الغربية، مصر والتى تبلغ مساحتها 33760 هكتار (وتقع

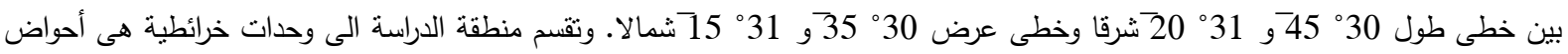
فيضية- شرفات نهرية عالية- شرفات نهرية متوسطة- شرفات نهرية منخفضة. وقد تم اختيار 9 قطاعات لتمنل منطقة الدراسة بالاضافة الى 18

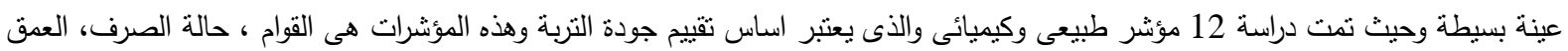
الفعال، مادة الاصل، فتات الصخور ، منحدر الميل، التوصيل الهيدروليكى، السعة الاحتفاظية بالماء، التوصيل الكهربى، رقم حموضة التربة، نسبة الصوديوم المنبادل ومحتوى التربة من كربونات الكالسيوم. وبناء على نتائج هذه التحليلات اتضح ان منطقة طنطا تم تقسيمها الى مستوى واحد تبعا لجودة المؤشرات الطبيعية( المنوسط) بينما اظهرت مؤشرات الجودة الكيميائية انه يوجد رتبتين ( المرتفع والمتوسط). أكدت النتائج ان أكثر من 44\% من اراضى طنطا متوسطة الجودة بينما 55.51\% تعتبر اراضى منخفضة الجودة تبعا لمؤشرات التربة الطبيعية والكيميائية. وانخفاض جودة التربة يسود على خواص المنطقة بانخفاض العمق وسوء الصرف والتوصيل الهيدروليكى. ويوصى البحث بأهمية ادارة التربة من خلال الممارسات بحيث تتوافق مع خواص التربة. 